\title{
HEAVY ROTATING STRING IN A CASING*
}

\author{
в Y CHIEN-HENG WU† (University of Minnesota)
}

1. Introduction. It is well known in the linear theory that a heavy string with one endpoint free has eigenfunctions of lateral displacement only at certain eigenvelocities of rotation $\omega_{n}$ which form a discrete spectrum. Kolodner [1] has shown that according to the more accurate non-linear theory, a string can rotate at any velocity $\omega>\omega_{1}$, and that for each $\omega$ in the range $\omega_{n}<\omega \leq \omega_{n+1}$ there are exactly $n$ distinct modes of rotational displacement. It has been found in [2] that even if the linear equation is used, a string can still rotate at any velocity $\omega>\omega_{1}$ if it is contained in a casing of small radius. Furthermore, for each $\omega$ in the range $\omega_{n} \leq \omega<\omega_{n+1}$ there are exactly $n$ distinct modes of rotation. The essential feature of this phenomenon is that the string is subjected to a constraint condition giving rise to a moving boundary. This result will be demonstrated by the two theorems proved in this paper.

2. Formulation. Consider the rotation of a string of length $L$ with its upper end $(x=L)$ fixed and lower end $(x=0)$ free. If we let $y(x)$ be the steady state solution for lateral displacement, then

$$
\frac{d}{d x}\left(\rho g x \frac{d y}{d x}\right)+\rho \omega^{2} y=0
$$

is the linearized governing equation in which $\rho$ is the density per unit length of the string and $\omega$ the angular velocity. Introducing $\xi=x / L, \eta=y / L, \Omega^{2}=\omega^{2} L / g$ we get

$$
\frac{d}{d \xi}\left(\xi \frac{d \eta}{d \xi}\right)+\Omega^{2} \eta=0 .
$$

This equation has a singularity at $\xi=0$. Therefore, appropriate boundary conditions for the eigenvalue problem are

$$
\eta(1)=0, \quad \eta(0) \text { finite }
$$

The eigenvelocities and mode shapes corresponding to equations (1) and (2) are $\Omega_{n}$ and $J_{0}\left(2 \Omega_{n} \xi^{1 / 2}\right)$ where $2 \Omega_{n}$ is the $n$th zero of $J_{0}(Z)$.

If the string is constrained to rotate in a casing of dimensionless radius $r / L=\delta \ll 1$, then the boundary conditions (2) become

$$
\eta(1)=0, \quad|\eta(\xi)| \leq \delta \ll 1 \quad(0 \leq \xi \leq 1)
$$

It will be shown that equations (1) and (3) have nontrivial solutions for any $\Omega>\Omega_{1}$. The existence of such solutions may easily be seen from the following auxiliary initial value problem.

We consider the function $u(\xi, a, \mu)$ on a real $\xi$ interval, $I_{\xi}=(\xi ; a \leq \xi \leq b<\infty)$, $0<a<1$, and a real $\mu$ interval $I_{\mu}=\left(\mu ; \Omega_{1}^{2} \leq \mu \leq M<\infty\right)$ such that 


$$
\begin{aligned}
\left(\xi, u(\xi, a, \mu), u^{\prime}(\xi, a, \mu)\right) \varepsilon D,\left(\xi \varepsilon I_{\xi},^{\prime}=\frac{\partial}{\partial \xi}\right), \\
\left(\xi u^{\prime}\right)^{\prime}+\mu u=0, \\
u(a, a, \mu)=\delta, \\
u^{\prime}(a, a, \mu)=0 .
\end{aligned}
$$

We now state the following theorem for the above defined initial value problem: Theorem 1. (1) $u(\xi, a, \mu)$ exists, is unique, and depends continuously on $a$ and $\mu$. (2) $u(\xi, a, \mu)$ has an infinite number of isolated zeros $Z_{n}(a, \mu), a<Z_{1}<$ $Z_{2}<\cdots<Z_{n}$ and $\lim _{n \rightarrow \infty} Z_{n}(a, \mu)=\infty$; furthermore, $Z_{n}(a, \mu)$ is a monotonically decreasing function of $\mu$ for constant $a$.

(3) If we define $\mu_{n}(a)$ to be such that $Z_{n}\left(a, \mu_{n}(a)\right)=1$, then there are an infinite number of $\mu_{n}, \mu_{1}(a)<\mu_{2}(a)<\cdots<\mu_{n}(a)$ and $\lim _{a \rightarrow 0} \mu_{n}(a)=\Omega_{n}^{2}, \lim _{a \rightarrow 1} \mu_{n}(a)=\infty$.

(4) $u_{n}(\xi, a)=u\left(\xi, a, \mu_{n}(a)\right)$ has exactly $n$ zeros in the interval $[a, 1]$ and $\max \left|u_{n}(\xi, a)\right|=u_{n}(a, a)=\delta$.

(5) The $\mu_{n}(a)$ are differentiable functions of $a$ and $d \mu_{n} / d a>0$; furthermore, $\lim _{a \rightarrow 1}$ $d \mu_{n} / d a=\infty$.

Proof: (1) Existence and uniqueness of $u(\xi, a, \mu)$.

Rewrite equation (4) as

$$
u^{\prime \prime}=-\frac{1}{\xi}\left(u^{\prime}+\mu u\right)=f\left(\xi, u, u^{\prime}, \mu\right) .
$$

Let $D_{\mu}$ be the domain of $\left(\xi, u, u^{\prime}, \mu\right)$ space

$$
D_{\mu}:\left(\xi, u, u^{\prime}\right) \varepsilon D \quad \mu \varepsilon I_{\mu}
$$

then $f$ is continuous on $D_{\mu}$ and

$$
\begin{aligned}
\left|f\left(\xi, u, u^{\prime}, \mu\right)-f\left(\xi, u_{1}, u_{1}^{\prime}, \mu\right)\right| & \\
& \leq \frac{\mu}{\xi}\left(\left|u-u_{1}\right|+\left|u^{\prime}-u_{1}^{\prime}\right|\right) \leq \frac{M}{a}\left(\left|u-u_{1}\right|+\left|u^{\prime}-u_{1}^{\prime}\right|\right)
\end{aligned}
$$

since $\mu \geq \Omega_{1}^{2}>1$. Thus $f$ satisfies a Lipschitz condition in $u$ and $u^{\prime}$ uniformly with respect to $\mu$ on $D_{\mu}$ and Part (1) of Theorem 1 follows from [3].

(2) Zeros of $u(\xi, a, \mu)$ :

A zero of a nontrivial solution of equation (4) is isolated. Indeed, let the solution $u$ vanish at $\xi_{0}$. Then $u^{\prime}\left(\xi_{0}\right) \neq 0$, for otherwise $u^{\prime \prime}$ and hence $u^{(k)}$ vanish at $\xi_{0}$ for all $k$ and $u \equiv 0$. This proves that $\xi_{0}$ is an isolated zero.

The unique solution of equations $(4,5,6)$ can be expressed explicitly in terms of Bessel functions

$$
u(\xi, a, \mu)=\left[J_{1}\left(2 \mu^{1 / 2} a^{1 / 2}\right) Y_{0}\left(2 \mu^{1 / 2} \xi^{1 / 2}\right)-Y_{1}\left(2 \mu^{1 / 2} a^{1 / 2}\right) J_{0}\left(2 \mu^{1 / 2} \xi^{1 / 2}\right)\right] \mu^{1 / 2} a^{1 / 2} \pi \delta .
$$

Since the Bessel functions have an infinite number of zeros, it is clear that $u$ has an infinite number of zeros at $\xi=Z_{n}(a, \mu)$ and $\lim _{n \rightarrow \infty} Z_{n}(a, \mu)=\infty$.

(3) $Z_{n}(a, \mu)$ decreases monotonically with $\mu$ for constant $a$.

If we let

$$
\Delta(\xi, a, \mu)=J_{1}\left(2 \mu^{1 / 2} a^{1 / 2}\right) Y_{0}\left(2 \mu^{1 / 2} \xi^{1 / 2}\right)-Y_{1}\left(2 \mu^{1 / 2} a^{1 / 2}\right) J_{0}\left(2 \mu^{1 / 2} \xi^{1 / 2}\right)
$$


then, by definition of $Z_{n}$ and equation (11), $\Delta_{a}\left(Z_{n}, \mu\right)=\Delta\left(Z_{n}, a, \mu\right)=0$. Since $u(\xi, a, \mu)$ depends continuously on its arguments so does $\Delta_{a}\left(Z_{n}, \mu\right)$. We may now formally differentiate $\Delta_{a}\left(Z_{n}, \mu\right)$ to get

$$
\left(\frac{\partial Z_{n}}{\partial \mu}\right)_{a}=-\frac{\partial \Delta_{n}}{\partial \mu} / \frac{\partial \Delta_{n}}{\partial Z_{n}}=\frac{Z_{n}}{\mu}\left\{\left[\frac{J_{0}\left(2 \mu^{1 / 2} Z_{n}^{1 / 2}\right)}{J_{1}\left(2 \mu^{1 / 2} a^{1 / 2}\right)}\right]^{2}-1\right\} .
$$

For $\mu$ large enough, we may approximate Bessel functions by the first term of their asymptotic expansions and the zeros of equation (8) are approximately

$$
2 \mu^{1 / 2} Z_{n}^{1 / 2} \simeq 2 \mu^{1 / 2} a^{1 / 2}+\frac{2 n-1}{2} \pi ;
$$

thus,

$$
\left|\frac{J_{0}\left(2 \mu^{1 / 2} z_{n}^{1 / 2}\right)}{J_{1}\left(2 \mu^{1 / 2} a^{1 / 2}\right)}\right| \simeq\left(\frac{a}{Z_{n}}\right)^{1 / 4}<1
$$

since $Z_{n}>a$. It follows that $\left(\partial Z_{n} / \partial \mu\right)_{a}<0$ for $\mu$ large. However, since $Z_{n}$ are the roots of $\Delta(\xi, a, \mu)=0,\left(\partial Z_{n} / \partial \mu\right)_{a}$ can never vanish unless $\mu=\infty$. Thus, $\left(\partial Z_{n} / \partial \mu\right)_{a}<0$ must hold for all values of $\mu$. This proves that $Z_{n}(a, \mu)$ is a monotonically decreasing function of $\mu$ for constant $a$.

(4) $\mu_{n}(a)$ and $\lim _{a \rightarrow 0} \mu_{n}(a)=\Omega_{n}^{2}, \lim _{a \rightarrow 1} \mu_{n}(a)=\infty$ :

Since there are an infinite number of $Z_{n}(a, \mu)$, it is clear that an infinite number of $\mu_{n}$ with $Z_{n}\left(a, \mu_{n}(a)\right)=1$ (or $\Delta\left(1, a, \mu_{n}(a)\right)=0$ ) exist. Furthermore, since $Z_{1}(a, \mu)<$ $Z_{2}(a, \mu)<\cdots<Z_{n}(a, \mu)$ and $\left(\partial Z_{n} / \partial \mu\right)_{a}<0$, the $\mu_{n}(a)$ must have the relation $\mu_{1}(a)<$ $\mu_{2}(a)<\cdots<\mu_{n}(a)$

To determine the limits of $\mu_{n}(a)$ as $a$ approaches zero, we note that $\mu_{n}(a)$ satisfies $\Delta\left(1, a, \mu_{n}(a)\right)=0$. Therefore, if $\mu_{n}^{0}=\lim _{a \rightarrow 0} \mu_{n}(a)$, we must have

$$
\lim _{a \rightarrow 0} \Delta\left(1, a, \mu_{n}(a)\right)=J_{0}\left(2 \mu_{n}^{01 / 2}\right)=0 ;
$$

thus,

$$
\lim _{a \rightarrow 0} \mu_{n}(a)=\mu_{n}^{0}=\Omega_{n}^{2} .
$$

Similarly, if $\mu_{n}^{1}=\lim _{a \rightarrow 1} \mu_{n}(a)$, we must have

$$
\lim _{a \rightarrow 1} \Delta\left(1, a, \mu_{n}(a)\right)=-1 / \pi\left(\mu_{n}^{1}\right)^{1 / 2}=0 ;
$$

thus,

$$
\lim _{a \rightarrow 1} \mu_{n}(a)=\mu_{n}^{1}=\infty .
$$

(5) $u_{n}(\xi, a)=u\left(\xi, a, \mu_{n}(a)\right)$ has $n$ zeros in $[a, 1]$ and $\max \left|u_{n}(\xi, a)\right|=\delta$.

By definition, $\mu_{n}(a)$ satisfies $Z_{n}\left(a, \mu_{n}(a)\right)=1$. It is clear that $u_{n}(\xi, a)$ has exactly $n$ zeros in the interval $[a, 1]$. These zeros are $Z_{n i}(a)=Z_{i}\left(a, \mu_{n}(a)\right)$ where $i=1,2,3, \cdots, n$. Explicitly:

$$
u_{n}(\xi, a)=\left[J_{1}\left(2 \mu_{n}^{1 / 2} a^{1 / 2}\right) Y_{0}\left(2 \mu_{n}^{1 / 2} \xi^{1 / 2}\right)-Y_{1}\left(2 \mu_{n}^{1 / 2} a^{1 / 2}\right) J_{0}\left(2 \mu_{n}^{1 / 2} \xi^{1 / 2}\right)\right] \mu_{n}^{1 / 2} a^{1 / 2} \pi \delta .
$$

This solution holds only for $a>0$. When $a=0$ the solution is $\delta J_{0}\left(2 \Omega_{n} \xi^{1 / 2}\right)$.

Comparing $u_{n}(\xi, a)$ with the equality 


$$
Y_{0}(z) J_{1}(z)-J_{0}(z) Y_{1}(z)=2 / \pi z,
$$

we find that $u_{n}(\xi, a)=\delta$ if and only if $\xi=a$. It follows that $u_{n}(\xi, a)<\delta$ for $a<\xi<1$ since $u_{n}(1, a)=0$.

(6) $\mu_{n}(a)$ are differentiable functions of $a$ and $d \mu_{n} / d a>0$.

By definition of $\mu_{n}(a)$, we have $\Delta_{1}\left(a, \mu_{n}(a)\right)=\Delta\left(1, a, \mu_{n}(a)\right)=0$. Formally differentiating $\Delta_{1}\left(a, \mu_{n}(a)\right)$, we get

$$
\frac{d \mu_{n}}{d a}=-\frac{\partial \Delta_{1}}{\partial a} / \frac{\partial \Delta_{1}}{\partial \mu_{n}}=\frac{\mu_{n}}{a} /\left\{\left[\frac{J_{1}\left(2 \mu_{n}^{1 / 2} a^{1 / 2}\right)}{J_{0}\left(2 \mu_{n}^{1 / 2}\right)}\right]^{2}-1\right\} .
$$

Since $\mu_{n}$ and $a$ must satisfy the equation $\Delta\left(1, a, \mu_{n}(a)\right)=0$, the denominator of the last equation never vanishes and hence $d \mu_{n} / d a$ exists. To prove $d \mu_{n} / d a>0$, we use the same argument as in the proof of part (2). For $\mu_{n}$ large enough, equation $\Delta\left(1, a, \mu_{n}(a)\right)=0$ may be approximated by the first term of its asymptotic expansion to give

$$
2 \mu_{n}^{1 / 2} \simeq 2 \mu_{n}^{1 / 2} a^{1 / 2}+\frac{2 n-1}{2} \pi
$$

thus,

$$
\left|\frac{J_{1}\left(2 \mu_{n}^{1 / 2} a^{1 / 2}\right)}{J_{0}\left(2 \mu_{n}^{1 / 2}\right)}\right| \simeq\left(\frac{1}{a}\right)^{1 / 4}>1
$$

since $a<1$. It follows that $d \mu_{n} / d a>0$ for $\mu_{n}$ large. However, $d \mu_{n} / d a$ can never vanish since $\mu_{n} \geq \Omega_{1}^{2} \neq 0$. Thus $d \mu_{n} / d a>0$ for all $a$. Furthermore, $\lim _{a \rightarrow 1} d \mu_{n} / d a=\infty$ since $\lim _{a \rightarrow 1} \mu_{n}(a)=\infty$ from part (3). We have thus completed the proof of part (5). The result of part (5), Theorem 1, is shown graphically in Fig. 1.

Theorem 2. The function $\eta(\xi)$ on the interval $(0,1)$ satisfying

$$
\begin{aligned}
& \left.\begin{array}{l}
\left(\xi \eta^{\prime}\right)^{\prime}+\Omega^{2} \eta=0 \\
\eta(1)=0, \quad|\eta(\xi)| \leq \delta
\end{array}\right\} \xi \varepsilon I_{\xi}^{\prime}(\xi ; \eta(\xi) \not \equiv \delta) \\
& \eta(\xi)=\delta \quad \xi \notin I_{\xi}^{\prime}
\end{aligned}
$$

has for any $\Omega_{n} \leq \Omega<\Omega_{n+1}$ exactly $n$ nontrivial solutions $\eta_{1}, \eta_{2}, \cdots, \eta_{n}$ such that

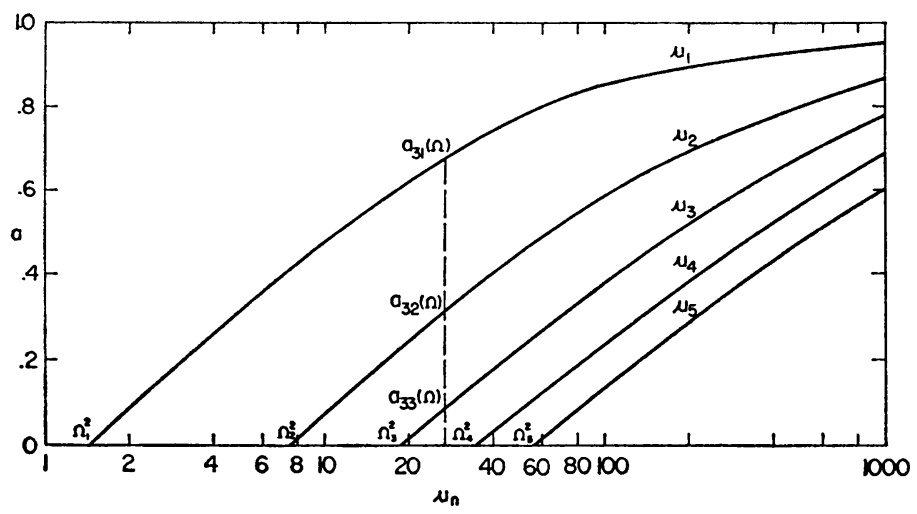

FIg. 1. 


$$
\eta_{i}(\xi)=\left\{\begin{array}{ll}
\delta & 0 \leq \xi \leq a_{n i} \\
u_{i}\left(\xi, a_{n i}\right) & a_{n i} \leq \xi \leq 1
\end{array} \quad(i=1,2, \cdots, n)\right.
$$

where $a_{n i}(\Omega)$ are determined by $\Omega^{2}=\mu_{i}\left(a_{n i}(\Omega)\right)$. The solution $\eta_{i}(\xi)$ has exactly $i$ isolated zeros.

Proof: From Theorem 1, the $\mu_{n}(a)$ are monotonically increasing functions of $a$ with the properties that $\mu_{1}(a)<\mu_{2}(a)<\cdots$ and $\lim _{a \rightarrow 0} \mu_{n}(a)=\Omega_{n}^{2}$. Thus for $\Omega_{n} \leq \Omega<\Omega_{n+1}$, there are $n$ values of $a, a_{n 1}(\Omega)>a_{n 2}(\Omega)>\cdots>a_{n n}(\Omega) \geq 0$, such that $\Omega^{2}=\mu_{i}\left(a_{n i}(\Omega)\right)$, $i=1,2, \cdots, n$. Corresponding to each $a_{n i}$ there is a $u_{i}\left(\xi, a_{n i}\right)$ defined in $a_{n i} \leq \xi \leq 1$ satisfying equation (9) and the boundary conditions $u_{i}\left(a_{n i}, a_{n i}\right)=\delta$ and $u_{i}\left(1, a_{n i}\right)=0$; furthermore, $u_{i}^{\prime}\left(a_{n i}, a_{n i}\right)=0$ and $\max \left|u_{i}\left(\xi, a_{n i}\right)\right|=\delta$. Therefore

$$
\eta_{i}(\xi)=\left\{\begin{array}{ll}
\delta & 0 \leq \xi \leq a_{n i} \\
u_{i}\left(\xi, a_{n i}\right) & a_{n i} \leq \xi \leq 1
\end{array} \quad(i=1,2, \cdots, n)\right.
$$

are the solutions of equations (9) and (10). Since $u_{i}\left(\xi, a_{n i}\right)$ has exactly $i$ isolated zeros, $\eta_{i}(\xi)$ also has $i$ isolated zeros.

Let us take for example $\Omega_{3}<\Omega<\Omega_{4}$, Fig. 1. There are three possible values for $a$, i.e., $a_{31}(\Omega), a_{32}(\Omega)$ and $a_{33}(\Omega)$. The three possible modes, one corresponding to each value of $a$, are

$$
\eta_{i}(\xi)=\left\{\begin{array}{ll}
\delta & 0 \leq \xi \leq a_{3 i} \\
u_{i}\left(\xi, a_{3 i}\right) & a_{3 i} \leq \xi \leq 1
\end{array} \quad(i=1,2,3) .\right.
$$

Acknowledgement. The author is indebted to Professor Robert Plunkett and Professor William H. Warner who read the manuscript and offered many helpful criticisms. This research was supported by the American Chemical Society Grant PRF 1376-A2, University of Minnesota.

\section{REFERENCES}

1. I. I. Kolodner, Heavy Rotating String-A Nonlinear Eigenvalue Problem, Communications on Pure and Applied Mathematics, 8, 39i-408, 1955

2. C. H. Wu, Critical Speeds of a Rotating Drill Shaft in a Uniform Casting, Technical Report ACS-PRF Grant \#1376-A2, University of Minnesota, 1964

3. E. A. Coddington, and N. Levinson, Theory of Ordinary Differential Equations, McGraw-Hill, 1955 Section 7 , p. 22 